\title{
The Anatomage table: Differences in student ratings between initial implementation and established use
}

\author{
S. Fyfel , G. Fyfe $e^{1}$, D. Dye' \& H. Radley-Crabb ${ }^{2}$
}

\begin{abstract}
Introduction: Increased student numbers and limited cadaver/prosected materials in health courses at Curtin University have required reviewing the way in which we teach our students in laboratory classes. The Anatomage table, an interactive tablet for viewing life-size anatomical images, was introduced into first-year human biology classes in 2013 to replace cadaver materials.

Methods: Two student cohorts were surveyed in 2013 and 2014 on their perception of the usefulness of the Anatomage table and other anatomy resources to their learning. On a scale of $0-100$, students were asked to rate a range of anatomy learning tools, including the Anatomage table.

Results: Respondents $(2013, \mathrm{n}=333 ; 2014, \mathrm{n}=329)$ rated video/animations most useful for learning (77.8/100), followed by models (63.9/100), plastinates (58.4/100) and the Anatomage table (42.4/100), a pattern consistent across gender, use of digital devices and cohort year. In 2014, respondents rated the Anatomage table more favourably $(42.4 / 100)$ than in $2013(36.9 / 100)(p=0.022)$. The Anatomage table was rated most helpful for understanding relative sizes of organs but least helpful for using correct anatomical terminology. Qualitative data showed that in 2013, students were frustrated by screen-freezing problems and low-quality graphics, issues that were mostly addressed by 2014 . Across both years, student comments were positive regarding the 3D aspect, seeing organ sizes and relationships, the slice tool for cross-sections and avoiding the
\end{abstract}

1 Faculty of Health Sciences, Curtin University, Australia

2 School of Biomedical Sciences, Curtin University, Australia

Correspondence

Sue Fyfe

Adjunct Professor

Faculty of Health Sciences

Bentley Campus

Curtin University

Perth 6845

Australia

Ph: +61 412564519

Email: S.Fyfe@curtin.edu.au 
need for cadaver specimens. Students also liked the Anatomage structured activities and pre-set images but wanted more time to explore and less than eight students around the table at any one time. Students felt unprepared for using the Anatomage table, and the single touch capacity was limiting when working in groups.

Conclusions: These findings indicate that touch-screen technology needs careful curriculum design and training for both students and staff to optimise its usefulness for learning.

Keywords: anatomy, students, medical; student engagement; health sciences.

\section{Introduction}

Understanding the structure of the human body is important in developing a basis of clinical practice for health professional students. Cadaveric dissection has been the traditional method for learning anatomy, but limited access to cadaveric material, budgetary restrictions, increasing numbers of students and available new technologies have led to change (Choudhury \& Goldsmith, 2012; Estai \& Bunt, 2015). Digitised images, plastinated specimens and animation have become popular (Azer \& Azer, 2016; Moro, Stromberga, Raikos, \& Stirling, 2017; Vertemati et al., 2018). Anatomage tables use digitised images to provide a large-scale "tablet-like" interactive experience for learning anatomy. Anatomage (www.anatomage.com) was founded in 2004 as a 3D imaging company and has expanded from radiology and dental software into more generic anatomical software. The Anatomage table uses Invivo5 imaging software to provide photographic rendering from CT scan data, some amalgamated with accurate, textured surface models to produce 3D images. The screen has a resolution of 3960x1080, with LCD technology that provides high contrast in normally-lit teaching rooms. The screens can be operated by a single user with fingers or styluses.

The Anatomage table provides life-size digital images of the male and female body, which can be rotated, virtually sectioned, resized and labelled. Virtual scalpels allow sectioning of the image in any plane, and the sectioned body part can then be rotated to examine internal structures from the "cut" surface. Schematic images allow for body systems, such as the integumentary, skeletal, circulatory and gastro-intestinal systems, to be isolated or removed. Radiology and clinical data can be imported into the system, and there is also a database of high resolution sectional CT scans - although neither of these options were used in the setting reported in this paper.

The Anatomage table has been used for teaching pelvic floor musculoskeletal anatomy (Eickmeyer, Wertsch, Lewandowski, Hoagland, \& Braza, 2013) and clinically-advanced teaching, such as fluoroscopically guided injection of the lumbar spine, hip and sacroiliac joints (Colorado, Wertsch, Hoagland, \& Braza, 2013). Although many have researched online anatomy resource use with students (Choudhury \& Goldsborough, 2012; Johnson, Palmer, Burton, \& Brockhouse, 2013; Tworek, Jamniczky, Jacob, Hallgrimsson, \& Wright, 2013), the literature reporting undergraduate student perceptions of Anatomage table use in teaching is still limited (Brown et al., 2015; Custer \& Michael, 2015; Hutchins, 2017). 
Anatomage tables were first incorporated into Human Structure and Function 100 (HSF100), a core unit for all health science courses at Curtin University, in Semester 1, 2013. The unit changed its name to Human Structure and Function (HUMB1000) at the beginning of Semester 1, 2015. Enrolments in HUMB1000 are large (around 2000 students in semester 1 and around 500 in semester 2 each year). HUMB1000 aims to ensure that students learn basic human biology across most body systems.

HUMB1000 is structured around a blended-learning model, where students access lectures through an iLecture system that captures Microsoft PowerPoint slides and audio and provides these and other electronic resources to students through the university's learning management system (LMS). A weekly 2-hour face-to-face workshop is facilitated by two tutors over the 12 -week semester. There is a maximum of 48 students per workshop class, and students work around a series of activity stations in groups of eight.

Human cadaver specimens had been used previously for these classes, but with increasing class sizes and demands on anatomy laboratory facilities for more advanced anatomy classes, an alternative approach was needed. Anatomage tables were identified as an option and incorporated into the unit practical classes. Figure 1 shows students using the Anatomage table as they undertake an activity during the class.

The Anatomage tables were incorporated into six of the 12 HUMB1000 workshops, with one Anatomage table provided in each classroom as one of the activity stations. Students were oriented to the Anatomage table in the first week of the semester and received detailed usage instructions in their weekly workshop material. Tutors received training in the use of the tables at their pre-semester orientation. In Semester 1, 2013, activities and materials were developed as the semester progressed, so there was no opportunity to pilot these activities and materials before they were used.

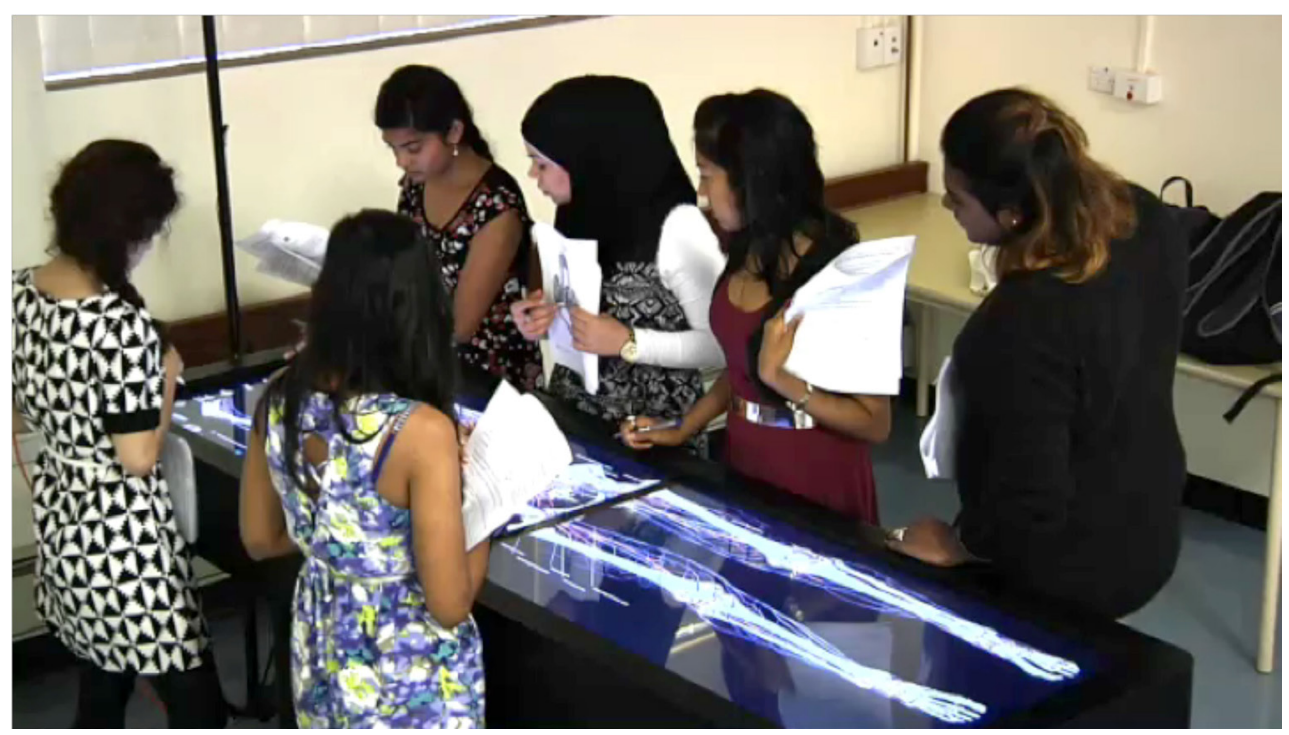

Figure 1. Students using Anatomage in HUMB1000. 
To ensure students were not disadvantaged by the replacement of cadaver materials with a technological alternative, it was important to evaluate the use of the Anatomage tables in light of other resources available to identify pros and cons of the technology for use in first-year human biology classes, to identify the best approach to developing materials and activities for their use and to determine how to integrate the tables into the classroom environment. Findings from the first students and tutors to use the Anatomage tables, in 2013, were used to modify learning activities and resources for the 2014 enrolment. In addition, new upgrades to the Anatomage graphics interface and software were purchased, and modifications to improve technical performance were made. Accompanying worksheets and activities were clarified, and tutor training was expanded.

An earlier paper described the initial implementation in 2013 (Fyfe, Fyfe, Dye, \& RadleyCrabb, 2013). This paper reports on 2014 data and compares data from the two cohorts.

\section{Methods}

HUMB1000 students were surveyed through an online Qualtrics questionnaire (http:// www.qualtrics.com) at the end of both Semester 1, 2013 and Semester 1, 2014. The Qualtrics system uses student emails to deliver the survey, but no name or email is connected with the data collected, allowing for an anonymous response. Students were provided with an information sheet about the project and an assurance that their participation was entirely voluntary, their response would be anonymous and no name or student ID would be connected with the survey data. All quantitative data were aggregated, and any quotes used were de-identified. Data were collected on demographic information, use of technology for learning and experiences and opinions about using learning resources, including the Anatomage table. Response choices including Likert scales, rating scales, text responses and radio buttons were used in the survey for ease of data collection and to provide a variety of response types. Students were asked to rate the usefulness of animation and videos, models, plastinates and the Anatomage table on a $0-100$ sliding scale. Using a Likert scale, they also rated the usefulness of the Anatomage table for learning anatomical terminology, understanding the relative size of organs and understanding the relationships between organs.

\section{Analysis}

Data were gathered in Qualtrics, and the 2013 and 2014 data sets, and a combined 2013/2014 data set, were then uploaded into SPSS for analysis (www.ibm.com/spss). Chi-square analysis and t-tests were used to determine if there were significant differences between group frequencies and means, and ANOVA was used to determine whether or not there were differences between multiple groups. Sample-size calculations for representativeness of each year sample were undertaken using the online Qualtrics calculator (http://www.qualtrics.com/blog/calculating-sample-size/). The significance level was set at 0.05 for all statistics. The denominator differs for different analyses because respondent numbers varied for different questions. 
Qualitative data were also collected through comment boxes on survey questions and with free-text response to four questions:

1. What were the best aspects of using the Anatomage table?

2. The worst aspects?

3. If you were designing an HSF100 practical session using the Anatomage table, what would you do?

4. Any other comments?

All comments were exported into a word document and manually categorised for main issues and ideas. Lead quotes were then identified for use in the text.

Ethical approval for the study was obtained from the Curtin University Human Research Ethics Committee.

\section{Results}

\section{Demographics}

Of the student enrolment in HUMB1000, 18\% responded to the online student survey in $2013(\mathrm{n}=333 / 1817)$ and $16.4 \%(\mathrm{n}=329 / 2003)$ in 2014 . The minimum numbers calculated to be representative of the full enrolment were 318 in 2013 and 323 in 2014. Thus, both samples (333 in 2013 and 329 in 2014) were representative of the full enrolment. Female respondents made up $80 \%$ of the sample, but this is representative of the whole population.

Whilst the total number of respondents to the overall survey was 333 in 2013 and 329 in 2014, not all students who responded to the survey responded to every question, and thus, there are different respondent numbers to a number of different questions. For example, only 326/333 (2013) and 306/329 (2014) respondents provided information on their gender and other demographic information. Demographic information is presented in Table 1 . In 2013, 85\% $(n=282 / 333)$ and in 2014, 32\% (105/329) of respondents made comments to the free-text questions.

Most respondents in both cohorts were young, novice learners experiencing their first semester of tertiary education. The mode and median age in both cohorts was 18 years. The average age of all respondents was 21.3 years (SD 6.9), with the 2013 cohort slightly younger (21.1 years, SD 6.8) on average than the 2014 group (22.1 years, SD 11.6). This difference was not significant $(p=0.2)$, and the overall distribution of ages did not significantly differ between the two groups $(p=0.11)$. No previous experience in tertiary study was reported by $70 \%$ of respondents.

\section{Use of technology}

Most respondents (90.7\%) in the 2013 cohort had a smart phone, and by 2014 , this had risen to $95.4 \%$ of the group; however, few students used anatomy apps on their phone, with no gender differences in either cohort or between 2013 and 2014. Tablet ownership also rose within the year but not significantly, with over half of both male 
and female students having a tablet or iPad in 2014. In 2013, 20\% of respondents accessed anatomy apps on their tablet to help them study for HUMB1000. However, only $7 \%$ used their tablet with anatomy apps in class, although the proportion of males was greater $(p=0.04)$, as shown in Table 1 . Using anatomy apps in class rose to $10 \%$ overall for the 2014 cohort, although this increase was not significant $(2013, p=0.28)$. A greater proportion of males used apps in class in 2014, but this difference was also not significant $(p=0.24)$.

Table 1

Demographic Characteristics and Technology Use, 2013 and 2014

\begin{tabular}{|c|c|c|c|c|c|c|c|}
\hline \multicolumn{8}{|c|}{ Demographic Characteristics 2013/2014 } \\
\hline \multirow[t]{2}{*}{ All $n=662$} & \multicolumn{3}{|c|}{2013} & \multicolumn{3}{|c|}{2014} & \multirow{2}{*}{$\begin{array}{c}2013 / 2014 \\
p^{*}\end{array}$} \\
\hline & $\begin{array}{c}\mathrm{F} \\
\mathrm{n}=284\end{array}$ & $\begin{array}{c}M \\
n=42\end{array}$ & All & $\begin{array}{c}F \\
n=254\end{array}$ & $\begin{array}{c}M \\
n=52\end{array}$ & All & \\
\hline \multirow[t]{2}{*}{$\begin{array}{l}\text { Average age } \\
\text { (SD) }\end{array}$} & $\begin{array}{l}21.25 \\
(7.04)\end{array}$ & $\begin{array}{l}20.59 \\
(5.76)\end{array}$ & $\begin{array}{l}21.08 \\
(6.79)\end{array}$ & $\begin{array}{r}21.21 \\
(7.1)\end{array}$ & $\begin{array}{c}23.19 \\
(7.4)\end{array}$ & $\begin{array}{c}22.08 \\
(11.61)\end{array}$ & 0.591 \\
\hline & $\%$ & $\%$ & $p$ chi sq & $\%$ & $\%$ & $p$ chi sq & $p$ chi sq \\
\hline Own a smart phone & 91.2 & 86.7 & 0.33 & 95.2 & 96.5 & 0.67 & 0.4 \\
\hline $\begin{array}{l}\text { Used anatomy apps } \\
\text { to help study }\end{array}$ & 14.7 & 10.3 & 0.46 & 14.1 & 15.2 & 0.64 & 0.41 \\
\hline Own a tablet/iPad & 44.4 & 33.3 & 0.17 & 55.4 & 54.4 & 0.89 & 0.39 \\
\hline $\begin{array}{l}\text { Used tablet/iPad } \\
\text { with anatomy apps }\end{array}$ & 19.7 & 21.4 & 0.88 & 19.9 & 22.4 & 0.98 & 0.87 \\
\hline $\begin{array}{l}\text { Used anatomy apps } \\
\text { on tablet in class }\end{array}$ & 5.7 & 8.3 & 0.04 & 9.1 & 16.1 & 0.24 & 0.28 \\
\hline
\end{tabular}

* $p$ values for differences between 2013/2014 cohorts

\section{Usefulness of anatomy resources}

Based on ratings on the sliding scale (0-100), students consistently rated animations and videos as the most useful resources to help them learn anatomical topics, followed by models, plastinates and, then, the Anatomage tables.

As shown in Table 2, between 2013 and 2014, there was a significant increase in the average rating for the usefulness of the Anatomage table, from 36.9\% (SD 28.2) in 2013 to $42.4 \%$ (SD 29.30) in $2014(p=0.022)$. There was little difference in ratings of usefulness between male and female respondents besides slightly lower ratings for plastinates by females $(p=0.22)$ and slightly higher ratings for the Anatomage table by males $(p=0.44)$ in 2013 . 
Table 2

Male and Female Student Ratings of the Usefulness of Anatomy Resources (on a 0-100 sliding scale)

\begin{tabular}{|l|c|c|c|c|c|c|c|}
\hline & \multicolumn{3}{|c|}{$\mathbf{2 0 1 3}$} & \multicolumn{3}{|c|}{$\mathbf{2 0 1 4}$} & \\
\hline & Females & Males & All & Females & Males & All & $\begin{array}{c}2013 / 2014 \\
p\end{array}$ \\
\hline Animation and videos & 78 & 77 & 78.1 & 77.7 & 79.3 & 77.8 & 0.86 \\
\hline Models & 62 & 66 & 63 & 64.2 & 63.1 & 63.9 & 0.49 \\
\hline Plastinates & 57 & 62 & 58 & 58.3 & 59.3 & 58.4 & 0.89 \\
\hline Anatomage tables & 37 & 33 & 36.9 & 42.6 & 41.8 & 42.4 & 0.022 \\
\hline
\end{tabular}

\section{Student ratings for Anatomage tables}

Table 3 shows that students rated Anatomage tables more useful for understanding the relative sizes of different organs and the relationships between organs in contrast with helping them use correct anatomical terminology. There were significant increases in evaluation of usefulness across all aspects from 2013 to 2014, especially for understanding of relationships between organs.

In the combined 2013/2014 sample, there was no difference between owning a tablet and the perception of how useful the Anatomage table was for understanding relationships between organs $(p=0.439)$, relative size of organs $(p=0.681)$ or anatomical terminology $(p=0.179)$. For those who did have a tablet $(\mathrm{n}=306)$, there was no significant difference between those who did or did not use anatomy apps on their tablet and how useful they felt the Anatomage table was for understanding relationships between organs ( $p=$ $0.218)$, relative size of organs ( $p=0.723)$ or anatomical terminology $(p=0.184)$.

Of those respondents who did have a tablet, a greater proportion used anatomy apps on their smartphones than those who did not own a tablet $(p=0.023)$. Although few students (14\%) used anatomy apps on their smartphones, there was no difference in ratings between those who did or did not use them on their smartphone and how useful they felt the Anatomage table was for understanding relative size of organs $(p=0.281)$ or anatomical terminology $(p=0.115)$. However, a difference was found between use of smartphone-based anatomy apps and perceived usefulness of the Anatomage table. Of those who used anatomy apps on their smartphone, $75.3 \%$ reported that the Anatomage table was helpful or very helpful for understanding the relationships between organs compared with $53.9 \%$ of those who didn't use anatomy apps on their smartphone $(p=0.051)$.

In $2013,70 \%$ of students reported insufficient time to use the tables and commented on problems with the table "freezing", being hard to control, having poor quality images and allowing only one person to interact with it at a time. Students commented that they didn't know how to use the Anatomage table or were not shown how to use it. 
I suspect that a lack of knowledge of what the table was capable of may have resulted in students not being very enthusiastic about using the Anatomage table. (Female, 2013) For [a] visual learner like myself, more time spent using the table might help in understanding the key concepts. (Female, 2013)

Table 3

Students' Views on Usefulness of the AnatomageTable

(on a 3-point Likert scale)

\begin{tabular}{l|c|c|c|c|}
\hline & & $\mathbf{2 0 1 3}$ & $\mathbf{2 0 1 4}$ & $\begin{array}{c}\boldsymbol{P} \\
(\mathbf{n = 3 3 3})\end{array}$ \\
Understanding the relative sizes of different organs & Mean & 2.22 & 2.32 & $\mathbf{2 0 1 3 / 2 0 1 4}$ \\
\hline Understanding the relationships between organs & SD & 0.76 & 0.70 & 0.090 \\
Helping use correct anatomical terminology & Mean & 1.79 & 1.97 & 0.002 \\
& SD & 0.78 & 0.72 & \\
& Mean & 1.65 & 1.83 & 0.004
\end{tabular}

Note: 1 = not very helpful, 2 = moderately helpful, 3 = very helpful

Almost $40 \%$ of the 2013 cohort reported that the table "hardly ever worked well in class", but this dropped to $21.8 \%$ in 2014 , a significant drop $(p<0.001)$ that was also reflected in very few comments in 2014 about problems with the table. In 2014, $73 / 112(65 \%)$ of teaching classes were facilitated by tutors who had also taught in 2013. Although the tutors' role was to facilitate and support the students learning and they did not present to the class using the Anatomage table, this familiarity and experience may also be a factor affecting students' perceptions of the Anatomage table.

The single-touch capacity of the table was often mentioned by students as a barrier for effective use. Single touch meant that paper, sleeves or hands of anyone but the current user would interfere with the operation of the table. Further, opportunities to learn how to use and/or engage with the Anatomage table were limited by the single-touch nature, as it was often the case that one student would control the table whilst others sat back and observed. Students commented that the sessions would have been better if everybody had a chance to use the table.

The worst aspect is working in large groups, meaning that not everyone can participate fully or as much as they would like. (Male, 2013)

It was too fast, and there was always one person that did everything for the rest of the table while the rest of us just sat and observed. (Female, 2013)

On the positive side, respondents in both cohorts reported that they liked the $3 \mathrm{D}$ aspect, seeing the sizes and relationships between organs, using the slice tool to see cross-sections and not having to see cadaver specimens. 
The life-size model was very helpful, as it helped me vision [sic] the anatomy of the human body more accurately. I was also able to see the intricacy of the organs as it was very detailed, which to me, is better than the synthetic models. (Female, 2013)

It was an interactive way of learning, a way that I found both interesting and effective in gaining an understanding of what was taught. (Male, 2013)

In summary, although other resources were rated as more useful than the Anatomage table, student perceptions of its helpfulness for their learning improved over the two semesters. The 2014 cohort had less concern about technical problems, with the visualisation of size and location of organs being the most highly-rated advantages.

\section{Discussion}

Most respondents were novice learners experiencing their first semester of tertiary education. Almost all had a smartphone and about half had a tablet, similar to that reported for Australian students surveyed in 2015 (Meyer, Stomski, Innes, \& Armson, 2016). Like Ellaway and colleagues (Ellaway, Fink, Graves, \& Campbell, 2014), our students tended to use their devices for personal study rather than in class. However in general, owning a smartphone or tablet did not affect student perceptions of Anatomage table usefulness. It was interesting, however, that those who had an iPad tended to use apps on their smartphone more than those who didn't, supporting findings from the 2014 Educause survey of over 10,000 university students in the US linking a positive attitude to technology with using digital resources across different platforms (Dahlstrom \& Bichsel, 2014).

Across all genders and cohorts, animations and videos were the most highly rated for usefulness, followed by models, plastinates and, then, the Anatomage table. For all students, understanding the sizes of organs and relationships between them were reported as the most useful aspects of the Anatomage table. Understanding organ relationships was also the most positive feature reported by Kazoka and Pilmane (2017), Hutchins (2017) and Custer and Michael (2015). Medical imaging students in the study by Custer and Michael (2015) reported that the "three-dimensional" dynamic nature of the images was most helpful in understanding organ relationships. The focus on understanding anatomical relationships was also highly ranked by medical students using online visually-based radiographic anatomy webpages (Marker, Juluru, Long, \& Magid, 2012).

Usefulness ratings of resources remained stable across both year groups, except that the Anatomage table's usefulness was rated significantly higher in 2014 . This may be because the 2013 cohort were the first group to use the table, when staff were still unfamiliar with the approach and were also learning how to best use it. Although there were staff who had not used the Anatomage table in 2014, most tutors had had experience with it in 2013 and may have had more confidence to assist students. Technical problems such as the table "freezing" were generally overcome, and improved image quality and labelling was available through a software upgrade in 2014. However, with only one person able to interact with the table at a time, issues relating to the spontaneity of 
response and interaction were still noted by the 2014 cohort. Because of this, perhaps eight students is too large a group around the table at one time; however, our results suggest conflicting views on the amount of direction, such as written questions or tableprogrammed settings, that students feel they need at the station. This may be related to the predominantly novice learners in our cohort compared with the postgraduate medical imaging students (Custer \& Michael, 2015) and the small number of nonfirst-year students involved in the study by Hutchins (2017). In addition, it appears that students in these studies had more time to spend using the Anatomage table, and those who spent more time were more positive about it (Custer \& Michael, 2015).

An earlier study of students using computer-based programmes for learning anatomy showed the critical factor for learning was student control of orientation (Garg, Norman, Spero, \& Maheshwari, 1999). When students are focused more on finding than exploring structures, in highly structured stations with limited time and limited user interaction, the Anatomage table may not be used to its best advantage. Visualisation is a key strategy for learning human biology (Pandey \& Zimitat, 2007), and intellectual effort is needed to link structures together in a three-dimensional manner, taking scale and function into account, for a deeper understanding. The way in which tutors assist students in this process may be the key to the successful implementation of any teaching resource. It may be that tutors who are less comfortable with technology and who have not necessarily used such resources for their own learning are less able to model learning approaches with the Anatomage table than they are with more traditional resources.

\section{Conclusion}

Our findings about student response to the Anatomage table has given us some insight into the factors that could make this resource more useful and meaningful for students and tutors, insights which may be useful for others too. Helping students and tutors feel more confident in using the table, structuring classes so that students have more opportunity to explore and control the table and giving more opportunities for smaller group interaction around the Anatomage table could continue to improve how students regard learning using this virtual anatomical resource.

\section{Acknowledgements}

The authors thank Mr Randy Strack for valuable contributions to this research, Michelle Broughton for organising and managing data collection and Curtin University students who participated in this project.

\section{References}

Azer, S. A., \& Azer, S. (2016). 3D anatomy models and impact on learning: A review of the quality of the literature. Health Professions Education, 2(2), 80-98.

Brown, J., Stonelake, S., Anderson, W., Abdulla, M., Toms, C., Farfus, A., \& Wilton, J. (2015). Medical students perception of Anatomage-3D interactive dissection table. International Journal of Surgery, 23, S17-S18. 
Choudhury, B., \& Goldsborough, I. (2012). The use of electronic media to develop transferable skills in science students studying anatomy. Anatomical Sciences Education, 5, 125-131.

Colorado, B., Wertsch, J., Hoagland, T., \& Braza, D. (2013). Teaching fluoroscopicallyguided injection procedures utilizing Anatomage. Paper presented at the 30th annual meeting of the American Association of Clinical Anatomists, Denver, Colorado. Retrieved from http://clinical-anatomy.org/images/downloads/Past_Meeting_ $\mathrm{PDF} /$ denver.pdf

Custer, T., \& Michael, K. (2015). The utilization of the anatomage virtual dissection table in the education of imaging science students. Journal of Tomography and Simulation, 1, 1-4.

Dahlstrom, E., \& Bichsel, J. (2014). ECAR study of undergraduate students and information technology. Retrieved from http://www.educause.edu/ecar

Eickmeyer, S., Wertsch, J., Lewandowski, L., Hoagland, T., \& Braza, D. (2013). Teaching pelvic floor musculoskeletal anatomy using Anatomage. Paper presented at the 30th Annual Meeting of the American Association of Clinical Anatomists, Denver, Colorado. Retrieved from http://clinical-anatomy.org/images/downloads/ Past_Meeting_PDFs/denver.pdf

Ellaway, R. H., Fink, P., Graves, L., \& Campbell, A. (2014). Left to their own devices: Medical learners' use of mobile technologies. Medical Teacher, 36, 130-138.

Estai, M., \& Bunt, S. (2016). Best teaching practices in anatomy education: A critical review. Annals of Anatomy, 208, 151-157. doi:10.1016/j.aanat.2016.02.010

Fyfe, G., Fyfe, S., Dye, D., \& Radley-Crabb, H. (2013). Use of Anatomage tables in a large first year core unit. Paper presented at the Electric Dreams 30th Ascilite Conference, Sydney, NSW.

Garg, A., Norman, G. R., Spero, L., \& Maheshwari, P. (1999). Do virtual computer models hinder anatomy learning? Academic Medicine, 74, S87-S89.

Hutchins, S. M. (2017). Are all organ systems created equal? Conference Proceedings: New Perspectives in Science Education (6th ed.), 276-279.

Johnson, I. P., Palmer, E., Burton, J., \& Brockhouse, M. (2013). Online learning resources in anatomy: What do students think? Clinical Anatomy, 26, 556-563.

Kazoka, D., \& Pilmane, M. (2017). Teaching and learning innovation in present and future of human anatomy course at RSU. Papers in Anthropology, 26(2), 44-52. doi:10.12697/poa.2017.26.2.05

Marker, D. R., Juluru, K., Long, C., \& Magid, D. (2012). Strategic improvements for gross anatomy web-based teaching. Anatomy Research International, 2012. doi:10.1155/2012/146262

Meyer, A. J., Stomski, N. J., Innes, S. I., \& Armson, A. J. (2016). VARK learning preferences and mobile anatomy software application use in pre-clinical chiropractic students. Anatomical Sciences Education, 9, 247-254. 
Moro, C., Štromberga, Z., Raikos, A., \& Stirling, A. (2017). The effectiveness of virtual and augmented reality in health science and medical anatomy. Anatomical Sciences Education, 10(6), 549-559. doi:10.1002/ase.1696

Pandey, P., \& Zimitat, C. (2007). Medical students' learning of anatomy: Memorisation, understanding and visualisation. Medical Education, 41, 7-14.

Tworek, J. K., Jamniczky, H. A., Jacob, C., Hallgrimsson, B., \& Wright, B. (2013). The LINDSAY Virtual Human Project: An immersive approach to anatomy and physiology. Anatomical Sciences Education, 6, 19-28.

Vertemati, M., Rizzetto, F., Vezzulli, F., Sampogna, G., Cassin, S., Canzato, F., \& Elli, M. (2018). Teaching anatomy in a modern medical course: An integrated approach at Vialba Medical School in Milan. MedEdPublish. doi:10.15694/ mep.2018.0000019.1 